\title{
面向社区生活圈构建的郊区居民社区依赖性研究 以上海市为例
}

\author{
傅行行 ${ }^{1,2}$, 申 悦 ${ }^{2,3^{*}}$ \\ (1. 南京大学建筑与城市规划学院, 南京 210093；2. 华东师范大学城市与区域科学学院,上海 200062; \\ 3. 华东师范大学中国现代城市研究中心, 上海 200062)
}

\begin{abstract}
摘 要: 在郊区化和社区生活圈构建的背景下, 提高郊区居民日常生活对社区空间的依赖性对于缓解城市发展压 力、提高居民生活质量和满意度有重要意义。论文将功能性依赖和情感性依赖 2 个维度相结合, 构建研究框架, 将 居民的社区依赖性划分为社区依赖、社区依靠、社区认同和社区排斥 4 种类型。以上海市为案例地区开展实证研 究, 基于 2017 年郊区居民行为调查的一手数据, 分析居民功能性依赖和情感性依赖的基本特征及其群体间差异, 并利用多项Logistics 回归模型, 研究社区空间特征对社区依赖性的影响。对不同群体和不同社区特征下社区依赖 性的模式进行总结, 以期为郊区社区生活圈规划提供研究依据。研究结果表明:不同群体的社区依赖性特征存在 显著差异, 小汽车拥有情况对于功能性依赖和情感性依赖的作用方向相反; 社区设施密度主要影响居民对社区的 功能性依赖; 社区绿地率对功能性依赖和情感性依赖都有显著的积极影响; 居民对社区的主观评价主要影响情感 性依赖。社区生活圈的规划应改善社区环境质量,合理配置绿地、开敞空间和休闲设施, 提高社区商业设施等级和 质量, 同时也要注重加强社会环境的建设。
\end{abstract}

关 键 词: 社区依赖性;社区生活圈;社区归属感;上海; 郊区

中国城市正在经历着深刻的发展转型,城市功 能转变、人口持续增长、快速郊区化等趋势对城市 建设和城市治理提出了新的要求。与此同时, “以 人为本” 成为城市研究和城市规划的重要指导思 想, 新型城镇化也越来越关注城市生活空间的构建 与居民生活质量的提升(宁越敏, 2012; 柴彦威等, 2014)。社区作为居民日常生活的基本空间单元， 逐渐成为城市研究与规划关注的重点, 近年来我国 许多城市先后提出以社区生活圈为基本空间单元 组织社区生活和配置公共资源, 例如, 上海市率先 提出全面构建 “15 分钟社区生活圈”,并发布了《上 海市 15 分钟社区生活圈规划导则(试行)》。
郊区化是中国大城市正在经历的最剧烈的变 化之一。在制度、经济、社会、规划等多种因素的综 合作用下, 郊区社区发展成为高度异质化的空间, 居民的生活空间发生重构, 生活质量亟待提升(张 艳等, 2013)。居住郊区化是郊区化最重要的表现 形式,但与此同时,分散中心城区的就业压力、交通 压力等也是郊区应发挥的职能, 就业活动和日常活 动的本地化乃至日常生活空间的郊区化才是郊区 居民理想的生活方式(申悦等, 2017)。因此, 提高郊 区居民日常活动对郊区空间的依赖性对于缓解大 城市中心城区发展压力、提高居民生活质量具有重 要意义。在这样的背景之下, 《上海城市总体规划

收稿日期 : 2018-12-11; 修订日期 : 2019-03-12。

基金项目: 国家自然科学基金项目(41871166,41601159); 上海市教育发展基金会和上海市教育委员会“晨光计划”项目(17CG20); 中央高校基本科研业务费项目(2018ECNU-QKT001)。[Foundation: National Natural Science Foundation of China, No. 41501180 and 41601159; "Chenguang Program" Supported by Shanghai Education Development Foundation and Shanghai Municipal Education Commission, No. 17CG20; Fundamental Research Funds for the Central Universities, No. 2018ECNU-QKT001. ]

第一作者简介:傅行行(1995-), 女, 浙江舟山人,硕士生, 主要从事城市地理学研究。E-mail: recherchefu@163.com *通信作者简介: 申悦(1987- ), 女, 上海人, 副教授, 主要从事城市地理学与行为地理学研究。E-mail: shenyue0519@163.com 
(2017-2035)》重点提出构建集就业、生活、服务等 功能为一体的郊区社区生活圈。

关注郊区居民日常活动对社区空间的依赖性， 有助于理解城市郊区发展的现状, 以及居民融人郊 区空间的情况, 对于构建郊区社区生活圈有重要的 意义(申悦等, 2018)。国内已有部分针对郊区生活 空间和活动空间开展的研究, 其中从时空间行为视 角出发, 研究个体对郊区空间的利用及其影响成为 重要的研究方向。例如, 申悦等(2013)利用 GPS 数 据刻画并分析了北京郊区居民的日常活动空间; 塔 娜等(2015)根据郊区居民的活动范围和出行频率对 居民的生活方式进行分类, 分析郊区化对居民日常 生活方式的影响。随着社区生活圈理论和实践探 索的逐渐深人, 一些研究开始从行为视角探讨社区 生活圈的规划对策。例如, 柴彦威等(2015)从居民 时空间行为分析出发, 构建了以 “基础生活圈一通 勤生活圈一扩展生活圈一协同生活圈”为核心的城 市生活圈规划理论模式; 李萌(2017)通过对上海市 部分居民的行为特征进行调查, 分析社区空间的供 需差异，提出构建社区生活圈的规划思路和对策; 孙道胜等(2017)基于居民个体活动的 GPS 数据, 划 分社区生活圈的圈层体系并提出公共服务设施的 空间优化策略。

社区生活圈的构建在注重居民对社区空间功 能性依赖的同时, 还需要关注居民对于社区的情感 依赖, 即居民的社区归属感。社区归属感是社会 学、心理学等领域研究的热点议题, 已有研究表明, 居民的社区归属感和生活满意度存在正相关性(何 深静等, 2014; 张景秋等, 2015)。环境心理学家将 人在心理上对某个地方产生的归属感称为“地方认 同”(Proshansky, 1978; 杨昀等, 2012), 相关研究多采 用量表测量法、观察法、案例研究等方法, 主要内容 包括地方认同的测度和影响因素(庄春萍等, 2011)。 研究表明, 影响地方认同的因素包括与个体自身相 关的社会经济特征(Proshansky et al, 1983)、与地方 相关的物质空间特征和社会文化特征等(庄春萍 等, 2011; 冯宁宁等, 2017), 以及居住时长等调节因 素(Stedman, 2006)。

已有关于社区依赖性的研究主要关注居民的 “社区认同”, 很少有研究将居民对社区的情感依赖 与其对社区空间的客观利用相结合, 而事实上, 居 民在功能上和情感上对社区空间的依赖是相辅相 成、缺一不可的。在地理学领域, 空间依赖是旅游
地理学和文化地理学研究的重要内容, 主要关注人 的主观情感,通常采用量表的形式衡量居民对某个 地方的认同或依恋程度 (白凯, 2010; 杜宗斌等, 2012; 熊帼等, 2013)。也有部分研究将居民对空间 的功能性依赖考虑在内 (Qian et al, 2011; 唐文跃, 2011; 王华等, 2014; 梁增贤等, 2016; Wu et al, 2019), 但是这些研究对功能性依赖的衡量通常也是通过 量表的形式由居民作答, 存在很强的主观性, 不能 很好地与情感性依赖相区分。从时空间行为的视 角出发, 分析个体的日常活动空间与社区空间的关 系, 能够更加客观地衡量居民对社区的功能性依 赖,与受访者汇报的通过量表测度的情感性依赖形 成 2 个不同的维度。将 2 个维度相结合能够相对综 合地反映居民的社区依赖性, 对现有研究形成补 充, 更加深人地体现社区生活圈构建的现实需求。

本文基于城市郊区化和社区生活圈规划两大 背景, 分别从功能性依赖和情感性依赖 2 个维度出 发,构建框架,研究郊区居民的社区依赖性。基于 上海市郊区居民行为调查的一手数据, 分析不同群 体功能性依赖和情感性依赖的基本特征, 结合功能 性依赖和情感性依赖 2 个维度的特征对居民的社区 依赖性进行分类,研究社区空间特征对居民社区依 赖性的影响,以期为郊区社区生活圈的构建提供研 究依据。

\section{1 研究设计}

\section{1 数据来源}

本文采用的数据来源于 2017 年华东师范大学 时空间行为研究团队开展的“上海市居民日常活动 与出行调查”。调查采取多阶段抽样方法, 在上海 市选择除崇明区以外的 8 个郊区行政区,结合空间 位置、区域发展以及社区类型多样性特征,分别选 取闵行区梅陇镇、宝山区顾村镇、嘉定区江桥镇、松 江区九亭镇、青浦区徐泾镇、奉贤区南桥镇、金山区 朱泾镇以及浦东新区的张江镇、周浦镇和南汇新城 镇为典型区域。在每个乡镇街道中结合社区类型 (商品房社区、售后公房社区、拆迁安置房社区、社 会保障房社区、城中村等)选取 3 7 个社区, 在最终 选取的 58 个社区中开展随机抽样以及社区人户问 卷调查(图 1)。

调查采取调查员半访谈式询问与手持移动设 备(平板电脑)相结合的形式, 对 1593 位居民进行了 


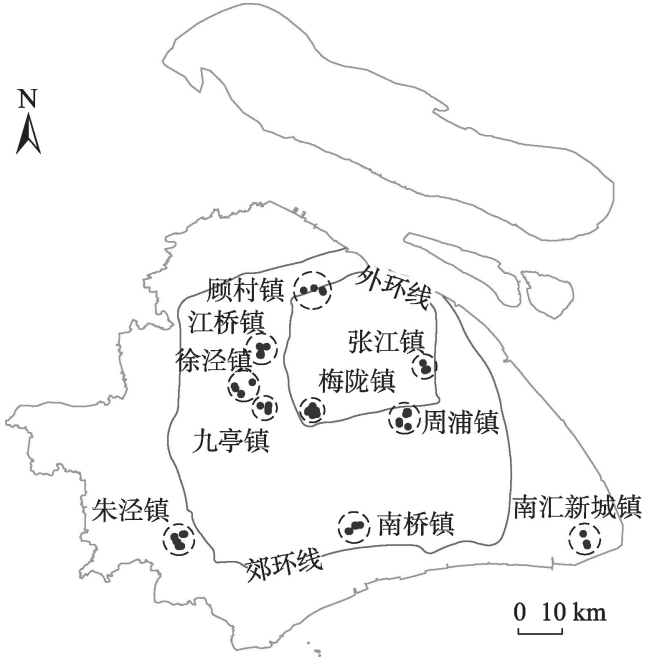

图 1 调查区域分布

Fig.1 The study areas

访问,记录了个体的社会经济属性、一个工作日和 一个休息日内的活动与出行情况、生活中的主观感 受和情感体验等内容。根据记录内容的完整性和 研究需要对调查样本进行校验和筛选, 最终选取有 效样本 1140 个, 问卷有效率为 $71.56 \%$ 。

\section{2 社区依赖性的研究框架}

黄向等(2006)在构建“场所依赖”的研究框架时, 将“场所依赖”解构为 “场所依靠” 和 “场所认同”两 个部分, “场所依靠”指功能性依赖, 即空间资源和 设施对个体活动的重要性, “场所认同”指精神性依 赖, 是个体在情感上与客观环境的依赖关系; 研究 采用一个二维坐标来表达 “场所依赖” 的结构,坐标 轴的两端分别表示场所依靠和场所认同, 坐标轴上 的位置表示功能性依赖和情感性依赖的分配比 例。Qian等(2014)在研究广州市外来移民的地方感 时, 也采取了类似的框架, 将地方感的建构划分成 情感依恋、认同形成和功能依赖 3 个维度。

本研究借鉴上述研究框架, 将居民对社区空间 的依赖性划分为功能性依赖和情感性依赖 2 个维 度。功能性依赖是指居民日常活动对社区空间的 利用程度, 通过居民家外非工作活动时长在社区内 外的分配来衡量 ${ }^{(1)}$ 。基于活动日志调查数据, 对居 民在连续的一个工作日和一个休息日内进行的家 外非工作活动时长进行统计, 居民对社区空间的利 用程度就等于居民在社区内进行家外非工作活动 的时长 (社区活动时长) 占家外非工作活动总时长的
比例。情感性依赖是指居民在心理上对社区空间 的依赖程度,通过问卷中的一个问题“对社区的归 属感如何” 来衡量, 用一个从 1 (非常没有归属感)到 5 (非常有归属感)的五级量表来测度。

根据功能性依赖和情感性依赖 2 个维度的特 征, 可以对居民的社区依赖性进行分类, 将其划分 为社区依赖、社区认同、社区依靠和社区排斥 4 种类 型(图 2)。如果居民对于社区的功能性依赖和情感 性依赖都比较强,则属于社区依赖型; 若居民对于 社区仅仅在情感上具有较强的归属感,但对于社区 空间的利用较少,则属于社区认同型; 若居民对于 社区空间的利用较多,但社区认同感和归属感较 弱,则属于社区依靠型; 如果居民对于社区的利用 较少, 且归属感也较弱, 则属于社区排斥型。

\section{2 不同群体社区依赖性的基本特征}

基于调查数据,对不同社会经济属性群体的社 区空间利用情况和社区归属感特征进行统计,分析 居民社区功能性依赖和情感性依赖的基本特征。

\section{1 不同群体的社区功能性依赖特征}

对不同群体的社区空间利用情况进行统计 (表 1),研究发现,样本总体社区内家外非工作活动 时长占家外非工作活动总时长的比例约为 $50 \%$, 反 映了郊区居民对社区空间利用的平均水平。

男性和女性的家外非工作活动时长差距较小, 但女性的社区活动时长明显多于男性, 即女性的日 常活动更依赖社区空间。不同年龄段居民对社区 空间利用的差异显著,随着年龄的增长, 居民对社 区空间的功能性依赖逐渐增强。上海户籍居民的 家外非工作活动时长多于外地户籍居民,但社区内

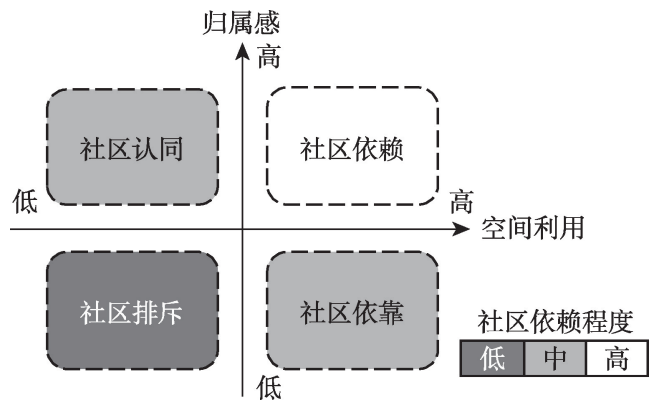

图 2 社区依赖的研究框架

Fig.2 Research framework of neighborhood attachment

(1) 研究将社区范围界定为居住地附近 $1 \mathrm{~km}$ 以内, 即步行约 $15 \mathrm{~min}$ 可达的范围。 
表 1 不同社会经济属性群体的社区空间利用情况

Tab.1 Usage of neighborhood space of different social groups

\begin{tabular}{|c|c|c|c|c|}
\hline 变量 & 样本数 & 家外非工作活动时长/min & 社区活动时长/min & $\begin{array}{c}\text { 社区活动时长/ } \\
\text { 家外非工作活动时长 }\end{array}$ \\
\hline 总体 & 1140 & 200.48 & 100.71 & $50.23 \%$ \\
\hline \multicolumn{5}{|l|}{ 性别 } \\
\hline 男性 & 575 & 199.22 & 94.16 & $47.26 \%$ \\
\hline 女性 & 565 & 201.76 & 107.28 & $53.17 \%$ \\
\hline \multicolumn{5}{|l|}{ 年龄 } \\
\hline 18 30 岁 & 333 & 194.26 & 81.22 & $41.81 \%$ \\
\hline 30 40 岁 & 334 & 205.82 & 90.30 & $43.87 \%$ \\
\hline 40 50岁 & 268 & 179.35 & 88.70 & $49.46 \%$ \\
\hline $50 \sim 60$ 岁 & 205 & 229.50 & 165.03 & $71.91 \%$ \\
\hline \multicolumn{5}{|l|}{ 户籍类别 } \\
\hline 上海户口 & 602 & 208.33 & 96.50 & $46.32 \%$ \\
\hline 外地户口 & 538 & 191.70 & 105.43 & $55.00 \%$ \\
\hline \multicolumn{5}{|l|}{ 就业情况 } \\
\hline 有稳定工作 & 891 & 195.29 & 84.75 & $43.40 \%$ \\
\hline 无稳定工作 & 249 & 219.04 & 157.82 & $72.05 \%$ \\
\hline \multicolumn{5}{|l|}{ 家庭平均月收人 } \\
\hline 5000 元及以下 & 166 & 175.01 & 123.14 & $70.36 \%$ \\
\hline $5001 \sim 10000$ 元 & 418 & 197.54 & 96.89 & $49.05 \%$ \\
\hline $10001 \sim 20000$ 元 & 410 & 205.89 & 100.16 & $48.65 \%$ \\
\hline 20000 元以上 & 146 & 222.67 & 87.67 & $39.37 \%$ \\
\hline \multicolumn{5}{|l|}{ 住房产权 } \\
\hline 住房自有 & 728 & 204.46 & 99.93 & $54.42 \%$ \\
\hline 住房租住 & 412 & 193.46 & 103.14 & $56.23 \%$ \\
\hline \multicolumn{5}{|l|}{ 家庭是否拥有小汽车 } \\
\hline 是 & 664 & 212.72 & 97.44 & $45.81 \%$ \\
\hline 否 & 476 & 183.41 & 105.28 & $57.40 \%$ \\
\hline
\end{tabular}

活动时长相对较少, 即外地户籍居民相对更加依赖 社区空间。没有稳定工作的居民社区内活动比例 显著高于有稳定工作的居民, 即没有稳定工作的居 民更加依赖社区空间。随着家庭月收人的增加, 社 区内活动比例降低, 即低收人家庭较为依赖社区空 间。家庭拥有小汽车的居民社区内活动比例明显 低于没有小汽车的居民, 即家中没有小汽车的居民 对社区空间更加依赖。

\section{2 不同群体的情感性依赖特征}

样本总体社区归属感的平均值为 3.54(表 2), 介 于 “一般” 和 “比较有归属感”之间。方差分析的结 果显示, 社区归属感在不同性别、年龄、户籍类别、 住房产权和家庭小汽车拥有情况群体之间表现出 显著的差异。女性样本的社区归属感高于男性, 年 龄越大的居民社区归属感越强, 即女性和中老年群 体在情感上更依赖社区空间。上海户籍居民社区
归属感显著强于外地户籍居民, 与已有研究结论一 致(吴蓉等, 2019)。家庭收人和社区归属感呈现倒 “U”形关系, 即中等收人群体社区归属感较强, 但这 种差异在统计上不显著。拥有住房产权和家庭拥 有小汽车的居民社区归属感较强, 对社区空间具有 更强的情感性依赖。

\section{3 社区依赖性类型及其影响因素}

根据前文提出的框架，对居民的社区依赖性类 型进行划分, 并以个体的社区依赖性类型为因变 量, 利用多项 Logistics 回归方法, 探讨在控制样本 社会经济属性的基础上,社区空间特征对社区依赖 性的影响。

\section{1 社区依赖性的类型划分}

已有研究指出, 在进行某项活动时, 如果选择 
表 2 不同社会经济属性居民的社区归属感

Tab.2 Sense of community of different social groups

\begin{tabular}{|c|c|c|c|}
\hline 变量 & 样本数 & 社区归属感 & $\begin{array}{c}\text { 单因素方差分析 } \\
F \text { 值 }\end{array}$ \\
\hline 总体 & 1140 & 3.54 & \\
\hline \multicolumn{4}{|l|}{ 性别 } \\
\hline 男性 & 575 & 3.49 & $1.976^{*}$ \\
\hline 女性 & 565 & 3.60 & \\
\hline \multicolumn{4}{|l|}{ 年龄 } \\
\hline 18 30 岁 & 333 & 3.43 & $3.709^{* * * *}$ \\
\hline 30 40 岁 & 334 & 3.56 & \\
\hline 40 50 岁 & 268 & 3.59 & \\
\hline $50 \sim 60$ 岁 & 205 & 3.65 & \\
\hline \multicolumn{4}{|l|}{ 户籍类别 } \\
\hline 上海户口 & 602 & 3.68 & $13.368^{* * * *}$ \\
\hline 外地户口 & 538 & 3.40 & \\
\hline \multicolumn{4}{|l|}{ 就业情况 } \\
\hline 有稳定工作 & 891 & 3.53 & 0.673 \\
\hline 无稳定工作 & 249 & 3.60 & \\
\hline \multicolumn{4}{|l|}{ 家庭平均月收人 } \\
\hline 5000 元及以下 & 166 & 3.49 & 1.171 \\
\hline $5001 \sim 10000$ 元 & 418 & 3.56 & \\
\hline $10001 \sim 20000$ 元 & 410 & 3.56 & \\
\hline 20000 元以上 & 146 & 3.53 & \\
\hline \multicolumn{4}{|l|}{ 住房产权 } \\
\hline 住房自有 & 728 & 3.65 & $40.207^{* * *}$ \\
\hline 住房租住 & 412 & 3.35 & \\
\hline \multicolumn{4}{|c|}{ 家庭是否拥有小汽车 } \\
\hline 是 & 664 & 3.61 & $3.625^{* * * *}$ \\
\hline 否 & 476 & 3.45 & \\
\hline
\end{tabular}

注: ***、***分别表示在 $0.1 、 0.05 、 0.01$ 的显著性水平下显著。

这一场所的可能性比选择其他场所的可能性更高, 那么对该场所就存在场所依赖(唐文跃, 2011), 即选 择该场所的可能性高于 $50 \%$ 就可认为存在功能性 依靠。因此, 本研究选取 50\%作为功能性依赖的划 分界限, 即社区内的家外非工作活动时长占家外非 工作活动总时长的比例高于 $50 \%$ 的样本功能性依 赖高; 低于或等于 $50 \%$ 或 2 天内未进行家外非工作 活动的样本功能性依赖低。在社区的情感性依赖 程度划分方面, 将社区归属感一般作为划分界限, 即社区归属感为 5(非常有归属感)或 4(比较有归属 感)的样本对社区有较强的归属感, 情感性依赖较 强; 社区归属感为 3 (一般)、2(比较没有归属感)或 1 (非常没有归属感)的样本对社区的归属感较弱, 即 情感性依赖较弱。
基于调查获取的数据, 对上海市郊区样本社区 依赖性的类型进行统计, 可以发现,社区认同型样 本比例最高, 社区依靠型样本比例最低(表 3$)$, 反映 了郊区居民对社区空间的利用程度总体较低,而社 区归属感总体较高。

\section{2 社区依赖性类型的模型构建}

研究采用多项 Logistics 回归方法, 以“社区排 斥型”为参照组, 在控制样本社会经济属性的前提 下分析社区空间特征对居民社区依赖性的影响。 社区空间特征包括社区的客观建成环境和居民的 主观感知特征 2 种类型, 其中客观建成环境特征由 社区访谈或基于 POI数据的空间分析获得, 相关变 量包括社区绿地率、公交车站可达性、社区商业设 施密度、社区休闲设施密度、社区住房类型; 居民的 主观感知特征通过问卷调查获得,包括居民对社区 环境健康程度、美观程度和安全程度的评价(表 4)。 作为控制变量的样本社会经济属性通过问卷调查 获得,包括年龄、性别、户籍类型、就业状况、家庭收 人、住房产权和家庭小汽车拥有情况。

\section{3 社区依赖性类型的影响因素分析}

模型拟合结果显示模型似然比检验显著性 $P<$ 0.001 , 模型拟合参数等于 0.210 , 因此模型具有一定 的解释力。

根据模型结果(表 5),社区空间特征对居民的社 区依赖性具有较为显著的影响。在客观建成环境 方面,社区绿地率对社区依赖、社区依靠和社区认 同 3 种类型都有显著的正影响, 即社区绿地率越高, 居民对社区的功能性依赖和情感性依赖都越强。 居住在商品房社区的居民更有可能属于社区依赖 或社区认同类型, 说明他们具有更强的社区归属 感,这与 Zhu等(2011)针对广州市的实证研究中所 发现的商品房社区居民的社区归属感高于其他类 型社区的结论相一致。社区的设施密度主要影响 社区依靠型, 反映了设施密度主要影响居民对于社 区的功能性依赖,而对情感性依赖的影响不显著。

表3 社区依赖性特征类型划分与统计

Tab.3 Types and statistics of neighborhood attachment

\begin{tabular}{llccc}
\hline 类型 & \multicolumn{1}{c}{ 社区空间利用 } & $\begin{array}{c}\text { 社区 } \\
\text { 归属感 }\end{array}$ & 频率 & 比例 \\
\hline 社区依赖 & $>50 \%$ & $>3$ & 205 & $18.0 \%$ \\
社区依靠 & $>50 \%$ & $\leqslant 3$ & 159 & $13.9 \%$ \\
社区认同 & $\leqslant 50 \%$ 及无家外非工作活动 & $>3$ & 459 & $40.3 \%$ \\
社区排斥 & $\leqslant 50 \%$ 及无家外非工作活动 & $\leqslant 3$ & 317 & $27.8 \%$ \\
\hline
\end{tabular}


表4 模型变量选取

Tab.4 Independent variables of the models

\begin{tabular}{|c|c|c|c|c|c|}
\hline 自变量 & 类型 & 备注 & 自变量 & 类型 & 备注 \\
\hline 社区特征 & & & 社会经济属性 & & \\
\hline 社区绿地率 & 连续 & & 年龄 & 连续 & \\
\hline 公交车站可达性 & 连续 & & 性别 & 分类 & $1=$ 男性 \\
\hline 社区商业设施密度 & 连续 & $1 \mathrm{~km}$ 路网缓冲区 & & & $0=$ 女性 \\
\hline 社区休闲设施密度 & 连续 & $1 \mathrm{~km}$ 路网缓冲区 & 户籍类型 & 分类 & $\begin{array}{l}1=\text { 上海户口 } \\
0=\text { 外地户口 }\end{array}$ \\
\hline 社区住房类型 & 分类 & $\begin{array}{l}1=\text { 商品房社区 } \\
0=\text { 非商品房社区 }\end{array}$ & 就业情况 & 分类 & $\begin{array}{l}1=\text { 有稳定工作 } \\
0=\text { 无稳定工作 }\end{array}$ \\
\hline 社区环境健康 & 有序 & $1=$ 非常不同意 & 家庭平均月收人 & 连续 & \\
\hline 社区环境美观 & & $\begin{array}{l}2=\text { =比较不同意 } \\
3=\text { 一般 }\end{array}$ & 住房产权 & 分类 & $\begin{array}{l}1=\text { 住房自有 } \\
0=\text { 住房租住 }\end{array}$ \\
\hline 社区环境安全 & & $\begin{array}{l}4=\text { 比较同意 } \\
5=\text { =非常同意 }\end{array}$ & 家庭小汽车拥有情况 & 分类 & $\begin{array}{l}1=\text { 有小汽车 } \\
0=\text { 无小汽车 }\end{array}$ \\
\hline
\end{tabular}

表 5 多项 Logistics 回归结果

Tab.5 Results of multiple logistics regression models

\begin{tabular}{|c|c|c|c|c|c|c|}
\hline & \multicolumn{2}{|c|}{ 社区依赖 } & \multicolumn{2}{|c|}{ 社区依靠 } & \multicolumn{2}{|c|}{ 社区认同 } \\
\hline & 概率比 & 显著性 & 概率比 & 显著性 & 概率比 & 显著性 \\
\hline \multicolumn{7}{|l|}{ 社区特征 } \\
\hline 社区绿地率 & 1.359 & 0.003 & 1.223 & 0.063 & 1.536 & $<0.001$ \\
\hline 公交车站可达性 & 1.070 & 0.586 & 1.030 & 0.818 & 1.114 & 0.295 \\
\hline 商品房社区 & 1.539 & 0.040 & 1.075 & 0.751 & 1.528 & 0.015 \\
\hline 社区商业设施密度 & 0.880 & 0.483 & 0.618 & 0.017 & 1.013 & 0.929 \\
\hline 社区休闲设施密度 & 1.237 & 0.196 & 1.492 & 0.028 & 0.920 & 0.543 \\
\hline 社区环境健康 & 1.891 & $<0.001$ & 1.131 & 0.461 & 1.811 & $<0.001$ \\
\hline 社区环境美观 & 1.285 & 0.133 & 1.126 & 0.478 & 1.361 & 0.026 \\
\hline 社区环境安全 & 0.942 & 0.731 & 0.859 & 0.381 & 0.910 & 0.514 \\
\hline \multicolumn{7}{|l|}{ 社会经济属性 } \\
\hline 年龄 & 1.310 & 0.011 & 1.379 & 0.004 & 1.070 & 0.449 \\
\hline 男性 & 0.739 & 0.126 & 0.719 & 0.112 & 0.691 & 0.024 \\
\hline 上海户口 & 2.342 & $<0.001$ & 1.634 & 0.047 & 2.192 & $<0.001$ \\
\hline 有稳定工作 & 0.421 & 0.001 & 0.582 & 0.043 & 0.855 & 0.494 \\
\hline 家庭平均月收人 & 0.890 & 0.280 & 0.920 & 0.370 & 0.725 & 0.006 \\
\hline 住房自有 & 1.242 & 0.387 & 0.915 & 0.726 & 1.419 & 0.084 \\
\hline 家庭有小汽车 & 0.989 & 0.958 & 1.113 & 0.614 & 1.478 & 0.024 \\
\hline 模型拟合参数: Cox \& S & & & & & & \\
\hline
\end{tabular}

其中商业设施密度较高的社区, 居民属于社区依靠 型的可能性更小, 而休闲设施密度较高的社区, 居 民属于社区依靠类型的可能性更大。

在居民的主观感知特征方面, 社区环境健康和 美观这 2 个评价指标对社区依赖性存在影响。社区 环境健康对社区依赖和社区认同都存在显著的正 影响, 说明健康的社区环境能够同时增强居民在功 能上和情感上对于社区的依赖性。社区环境美观
对社区认同存在积极影响, 即美观的社区环境能够 增强居民的社区归属感。Lu等(2018)对温州市门 禁社区的研究表明居民对社区外观的满意度越高, 社区依赖的程度越高, 而本文进一步揭示了这种影 响更多的是在情感方面产生的依赖性。

居民的社会经济属性与社区依赖性之间也存 在着显著的相关性。与前文基本统计所得到的结 论相似, 年龄越大的居民越可能是社区依赖和社区 
依靠型, 即对社区空间的功能性依赖随着年龄的增 长而增强, 情感性依赖也有一定的增加。男性居民 社区认同的可能性显著低于女性, 社区依赖和社区 依靠的可能性也较低(但在统计上不显著), 即女性 对社区空间的功能性依赖和情感性依赖都较强, 这 与 Lewicka(2005)以及吴蓉等(2019)的研究结论相 一致。上海户口居民社区依赖、社区依靠和社区认 同的可能性均高于外地户口居民, 反映了非户籍居 民比较容易承受社区排斥, 这与已有的理论和实证 研究结论相符合。有稳定工作的居民属于社区依 赖和社区依靠的可能性较低,即他们对社区空间的 利用较少。家庭收人较高的居民对社区空间的功 能性依赖和情感性依赖都比较弱, 根据已有研究, 这可能是由于高收人群体更强的流动性所导致的 (Lu et al, 2018)。住房自有和家庭有小汽车的居民 社区认同的可能性较大。

\section{4 居民社区依赖性的特征模式}

根据前文分析, 对居民社区依赖性的特征及其 影响因素进行总结, 分别得到不同社会经济属性群 体和不同社区空间特征对社区依赖性影响的模式图 (图 3、图 4), 并面向社区生活圈规划提出相关建议。

\section{1 社区依赖性的社会经济属性模式}

老年人、女性、无业者、低收人人群等弱势群体 对社区空间的功能性依赖较强。中老年居民和女 性群体在社区内的活动较多, 对远距离出行的偏好

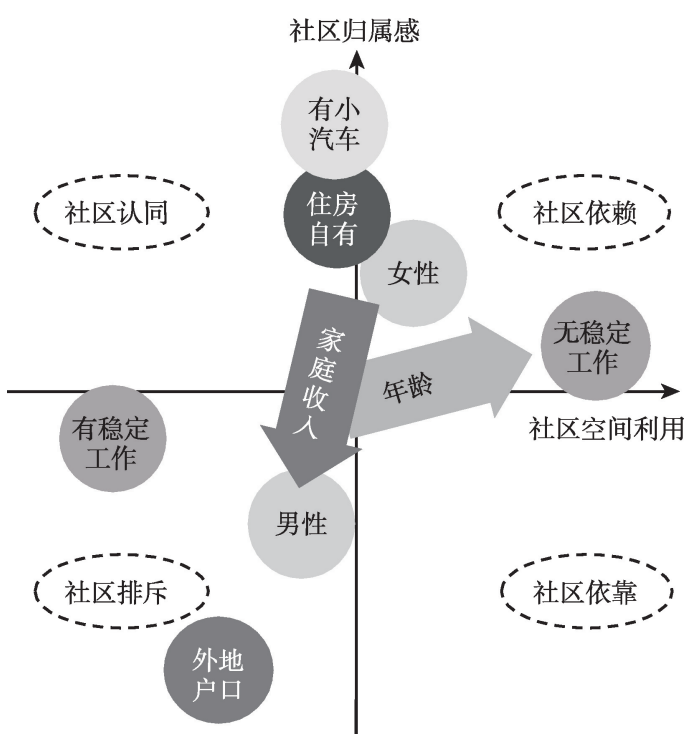

图 3 社区依赖性的社会经济属性模式

Fig.3 Neighborhood attachment and socioeconomic attributes
较低,因此他们对社区空间的功能性依赖较强。居 住在郊区的就业者通勤距离普遍较长, 由此形成的 时空制约会减少居民在社区内的活动 (申悦等, 2013; 刘志林等, 2015), 同时, 他们在工作地附近和 通勤途中活动的可能性也有所增加, 因此有稳定工 作的居民对社区空间的功能性依赖相对较弱。家 庭收人较低的居民圊于出行成本和交通可达性等 因素,在市中心、郊区次中心等区域的活动相对较 少, 日常活动主要集中在家附近,因此对社区空间 的功能性依赖较强。综上, 在社区规划中, 应着重 考虑这部分弱势群体的需求, 提高医疗、养老、就业 等各类服务水平, 同时也要考虑如何提高其他居民 对社区空间的功能性依赖。例如, 提高郊区的土地 利用混合程度, 推动就业的本地化, 促进职住均衡, 从而减轻过长的通勤距离对居民日常活动造成的 时空制约。

小汽车是郊区居民出行的主要方式之一,一方 面, 家庭有小汽车的居民出行更便利, 在郊区的生 活更舒适, 同时他们需要利用社区的停车设施, 容 易在心理上产生对社区空间的认同; 另一方面小汽 车的使用扩大了居民的活动空间范围, 降低了他们 对社区的功能性依赖。由此可见, 舒适便利的居住 条件能够提高居民对社区空间的情感性依赖,社区 生活圈规划应重点改善社区的公共服务水平,同时 需要完善郊区的公共交通系统,提高郊区居民出行 的便利性和居住的舒适度, 从而提高居民对社区的 情感性依赖。

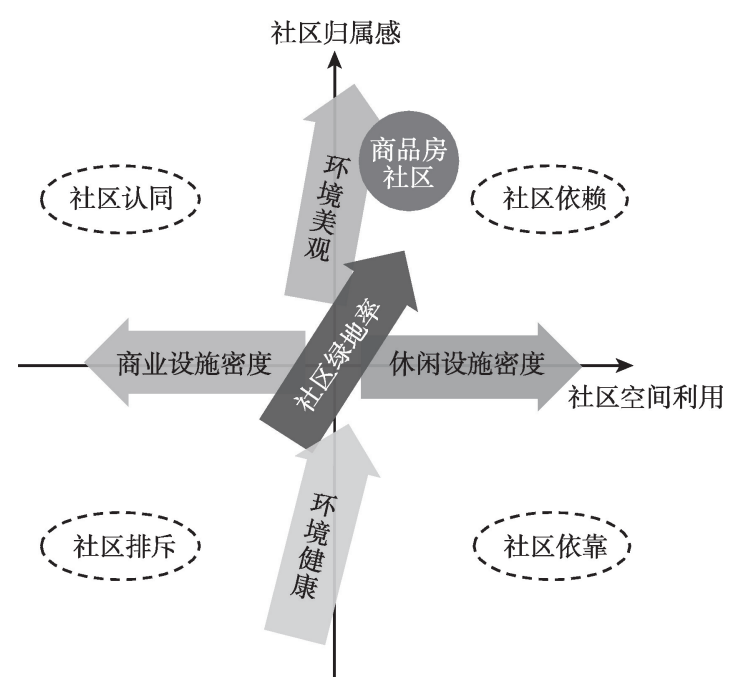

图 4 社区依赖性的社区特征模式

Fig.4 Neighborhood attachment and neighborhood characteristics 
外地户口居民对社区空间的依赖性尤其是情 感性依赖明显低于上海户口居民, 由于没有本地户 口, 他们能够获得的社会资本比较有限, 社会联系 也较少, 因此社区归属感较低。高收人家庭对居住 环境的衡量标准更高, 且他们与社区居民之间较难 建立紧密的联系, 因此对社区的认同感较低。拥有 住房产权的居民具有比较强的社区归属感, 主要是 由于他们的流动性较低, 在社区内的居住状态更加 稳定,因此对社区更容易产生情感性依赖。可见， 社区生活圈规划不仅要考虑社区的客观环境, 也要 考虑社区的社会环境, 鼓励居民参与社区活动, 促进 自下而上的社区治理,增强社区居民之间的联系， 建立良好的社区社会网络,促进社区的社会融合。

\section{2 社区依赖性的社区空间特征模式}

在社区空间特征对居民社区依赖性的影响方 面, 社区设施密度主要影响社区空间利用, 居民对社 区环境的主观评价主要影响社区归属感, 社区绿地 率对社区归属感和社区空间利用都有显著的影响。

社区休闲设施密度越高, 居民对社区空间的功 能性依赖越强, 社区休闲设施为居民提供日常游 憩、锻炼、社交等活动的场所, 因此能增加居民对社 区空间的利用。而社区商业设施密度越高, 居民对 社区空间的功能性依赖越弱, 这可能是由于两方面 原因所导致:一方面郊区社区的商业设施同质性较 强, 且大多数规模较小, 只能满足最基本的日常需 求; 另一方面, 居民到郊区居住主要是为了改善居 住环境, 他们对社区的开放空间、体育设施等有较 高的需求, 而社区商业设施密度高可能会使这类空 间相对减少。在社区生活圈的规划中, 应充分考虑 社区内的公园广场、体育设施等开敞空间的布置, 同时应对社区商业数量、规模和类型进行控制和引 导, 提升郊区商业质量, 使郊区商业设施对环境的 影响尽可能小, 最大程度地满足不同居民的需求。

社区绿地率能够提高居民对社区空间的功能 性依赖和情感性依赖, 社区绿地不仅能使社区环境 更加美观,也能为居民提供社交、运动等活动场所, 因此能够增加居民对社区空间的利用, 也能促进居 民产生对社区的情感认同。居住在商品房社区的 居民对社区空间的情感性依赖较强, 更为优越的居 住环境和便利的生活设施能够提高他们对社区的 归属感。选择居住在郊区的居民较为注重社区的 健康、舒适和美观程度, 因此健康、美观的社区环境 能够提高居民对社区空间的认同, 增强情感性依
赖。因此,社区生活圈的规划需要重视社区环境质 量的维持或改善,控制郊区社区的建筑密度和容积 率, 保证一定的社区绿地率, 注重社区景观及小规 模开敞空间的设置,使社区环境更加健康、美观。

\section{5 结论与讨论}

本研究聚焦郊区居民的社区依赖性, 将功能性 依赖和情感性依赖 2 个维度相结合, 构建研究框架, 对居民的社区依赖性进行分类。以上海市为案例 地区开展实证研究,分析居民社区依赖性的基本特 征及其群体间差异, 利用多项 Logistics 回归模型, 研究社区空间特征对居民社区依赖性的影响,并对 不同群体和不同社区特征下社区依赖性的模式进 行总结。研究结果表明:

(1) 不同群体的社区依赖性之间存在显著差 异:女性居民和上海户口居民对社区空间的功能性 依赖和情感性依赖均较强, 年龄较大的居民和没有 稳定工作的居民对社区空间的功能性依赖较强, 高 收人居民的社区依赖性较弱; 已有研究对性别、年 龄、户籍、收人、就业等因素关注较多,本研究结论 与已有结论基本一致 (Lewicka, 2005; 申悦等, 2013; 刘志林等, 2015; Lu et al, 2018; 吴蓉等, 2019)。本研究也揭示了小汽车在功能性依赖和情 感性依赖 2 个方面的复杂性作用, 即家庭有小汽车 的居民对社区的功能性依赖较弱,但有较强的社区 归属感; 已有研究对小汽车拥有情况的关注较少, 而本研究的案例地是郊区, 居民的日常出行更依赖 小汽车, 有必要考虑小汽车的影响, 而研究结论也 反映了将功能性依赖与情感性依赖作为 2 个维度分 别考虑的意义和重要性。

(2) 社区客观建成环境对居民的社区依赖性存 在显著影响:社区设施密度主要影响居民对社区的 功能性依赖, 社区休闲设施密度越高, 居民对社区 空间的功能性依赖越强, 而商业设施密度越高, 功 能性依赖越弱; 社区绿地率对功能性依赖和情感性 依赖都有显著的积极影响。与已有研究结论类似 (Zhu et al, 2011), 居住在商品房社区的居民具有较 强的社区归属感。

(3) 社区主观环境主要影响居民对社区的情感 性依赖: 居民对社区环境健康和美观程度的评价越 高, 社区归属感越强。已有研究表明, 对社区外观 的满意度越高, 社区依赖的程度就越高( Lu et al, 
2018), 本研究进一步揭示了对社区外观的满意度 主要影响居民对社区的情感性依赖。

相比于已有研究单纯考虑社区居民对社区的 情感认同或功能性依赖, 本研究将 2 个维度结合, 构 建了社区依赖性研究的综合性框架。研究结论对 于社区生活圈规划、提高郊区居民的生活质量与满 意度有一定的参考价值。郊区社区生活圈规划应 综合考虑社区物质环境和社会环境的构建。合理 安排用地, 引导居民就业本地化, 推动职住均衡; 改 善公共交通可达性, 提高开敞空间、休闲娱乐设施 的可达性; 合理配置社区内休闲设施, 提高社区商业 设施的等级和质量, 满足居民多样化需求; 重视社 区环境质量的维持或改善, 控制建筑密度, 保证绿地 率, 注重社区景观和公共空间的设置。社区生活圈 规划也要关注社区社会环境的构建, 提高居民的社 区参与度, 强化社区凝聚力, 促进社区社会融合。

\section{参考文献(References)}

白凯. 2010. 乡村旅游地场所依赖和游客忠诚度关联研究: 以西安市长安区“农家乐”为例 [J]. 人文地理, 25(4): 120125. [Bai K. 2010. A study on the relationship between place attachment and tourists' loyalty in rural tourism destinations. Human Geography, 25(4): 120-125. ]

柴彦威, 申悦, 陈梓烽. 2014. 基于时空间行为的人本导向的 智慧城市规划与管理 [J]. 国际城市规划, 29(6): 31-37, 50. [Chai Y W, Shen Y, Chen Z F. 2014. Towards smarter cities: Human- oriented urban planning and management based on space-time behavior research. Urban Planning International, 29(6): 31-37, 50. ]

柴彦威, 张雪, 孙道胜. 2015. 基于时空间行为的城市生活圈 规划研究: 以北京市为例 [J]. 城市规划学刊, (3): 61-69. [Chai Y W, Zhang X, Sun D S. 2015. A study on life circle planning based on space time behavioural analysis: A case study of Beijing. Urban Planning Forum, (3): 61-69. ]

杜宗斌, 苏勤, 姜辽. 2012. 社区参与对旅游地居民社区归属 感的中介效应: 以浙江安吉为例 [J]. 地理科学, 32(3): 329-335. [Du Z B, Su Q, Jiang L. 2012. Mediate effects of community involvement influence on sense of community in tourism destination: A case study of Anji County in Zhejiang Province. Scientia Geographica Sinica, 32(3): 329335. ]

冯宁宁, 崔丽娟. 2017. 从恢复体验到地方依恋: 环境偏好与 居住时长的作用 [J]. 心理科学, 40(5): 1215-1221. [Feng N N, Cui L J. 2017. The relation between restorative experience and place attachment: The role of environment preference and length of residence. Journal of Psychological
Science, 40(5): 1215-1221. ]

何深静, 齐晓玲. 2014. 广州市三类社区居住满意度与迁居 意愿研究 [J]. 地理科学, 34(11): 1327-1336. [He S J, Qi X L. 2014. Determinantd of relocation satisfaction and relocation intention in Chinese Cities: An empirical investigation on three types of residential neighborhood in Guangzhou. Scientia Geographica Sinica, 34(11): 1327-1336. ]

黄向, 保继刚, Wall Geoffrey. 2006. 场所依赖 (place attachment): 一种游㮩行为现象的研究框架 [J]. 旅游学刊, (9): 19-24. [Huang X, Bao J G, Wall G. 2006. Place attachment: A conceptual framework for understanding recreational behavior. Tourism Tribune, (9): 19-24. ]

李萌. 2017. 基于居民行为需求特征的 “ 15 分钟社区生活圈” 规划对策研究 [J]. 城市规划学刊, (1): 111-118. [Li M. 2017. The planning strategies of a 15-minute community life circle based on behaviors of residents. Urban Planning Forum, (1): 111-118. ]

梁增贤, 许德祺. 2016. 城市居民日常活动的社区依赖性研 究: 以深圳华侨城为例 [J]. 人文地理, 31(2): 29-35, 54 . [Liang Z X, Xu D Q. 2016. Daily activities and community dependence of urban residents: The case of Shenzhen overseas Chinese Town, China. Human Geography, 31(2): 29-35, 54. ]

刘志林, 廖露, 钮晨琳. 2015. 社区社会资本对居住满意度的 影响: 基于北京市中低收人社区调查的实证分析 [J]. 人 文地理, 30(3): 21-27, 71. [Liu Z L, Liao L, Niu C L. 2015. Residential satisfaction of community social capital: An empirical study of middle and low income residents in urban Beijing. Human Geography, 30(3): 21-27, 71. ]

宁越敏. 2012. 中国城市化特点、问题及治理 [J]. 南京社会科 学, (10): 19-27. [Ning Y M. 2012. A study on the urbanization, urban problems and solution in China. Social Sciences in Nanjing, (10): 19-27. ]

申悦, 柴彦威. 2013. 基于 GPS 数据的北京市郊区巨型社区 居民日常活动空间 [J]. 地理学报, 68(4): 506-516. [Shen Y, Chai Y W. 2013. Daily activity space of suburban megacommunity residents in Beijing based on GPS data. Acta Geographica Sinica, 68(4): 506-516. ]

申悦, 柴彦威. 2018. 基于日常活动空间的社会空间分异研 究进展 [J]. 地理科学进展, 37(6): 853-862. [Shen Y, Chai Y W. 2018. Progress of research on sociospatial differentiation based on daily activity space of urban residents. Progress in Geography, 37(6): 853-862. ]

申悦, 塔娜, 柴彦威. 2017. 基于生活空间与活动空间视角的 郊区空间研究框架 [J]. 人文地理, 32(4): 1-6. [Shen Y, Ta N, Chai Y W. 2017. Research framework of suburban space based on perspective of living space and activity space. Human Geography, 32(4): 1-6. ] 
孙道胜, 柴彦威. 2017. 城市社区生活圈体系及公共服务设 施空间优化: 以北京市清河街道为例 [J]. 城市发展研究, 24(9): 7-14, 25, 2. [Sun D S, Chai Y W. 2017. Study on the urban community life sphere system and the optimization of public service facilities: A case study of Qinghe area in Beijing. Urban Development Studies, 24(9): 7-14, 25, 2. ]

塔娜, 柴彦威, 关美宝. 2015. 北京郊区居民日常生活方式的 行为测度与空间-行为互动 [J]. 地理学报, 70(8): 12711280. [Ta N, Chai Y W, Kwan M P. 2015. Suburbanization, daily lifestyle and space- behavior interaction in Beijing. Acta Geographica Sinica, 70(8): 1271-1280. ]

唐文跃. 2011. 城市居民游憩地方依恋特征分析: 以南京夫 子庙为例 [J]. 地理科学, 31(10): 1202-1207. [Tang W Y. 2011. The characteristics of urban residents' place attachment to recreational block: A case study of Nanjing Confucius temple block. Scientia Geographica Sinica, 31(10): 1202-1207. ]

王华, 严婷. 2014. 城市居民休闲的地方依恋研究: 以广州荔 枝湾为例 [J]. 地域研究与开发, 33(5): 104-108. [Wang H, Yan T. 2014. Research on urban residents' place attachment to leisure places: A case of Guangzhou Litchi bay. Areal Research and Development, 33(5): 104-108. ]

吴蓉, 黄旭, 刘晔, 等. 2019. 广州城市居民地方依恋测度与 机理 [J]. 地理学报, 74(2): 1-15. [Wu R, Huang X, Liu Y, et al. 2019. Place attachment of urbanites in Guangzhou: Measurement and mechanism. Acta Geographica Sinica, 74 (2): 1-15. ]

熊帼, 张敏, 姚磊, 等. 2013. 大学生的地方依恋特征与形成机 制: 基于南京仙林大学城的调查 [J]. 人文地理, 28(5): 3135, 128. [Xiong G, Zhang M, Yao L, et al. 2013. Study on characteristics and formation mechanism of college students' place attachment: Based on the investigation of xianlin university town. Human Geography, 28(5): 31-35, 128. ]

杨昀, 保继刚. 2012. 旅游社区外来经营者地方依恋的特征 分析: 以阳朔西街为例 [J]. 人文地理, 27(6): 81-86. [Yang Y, Bao J G. 2012. The characteristics of non- local owners' place attachment in tourism community: A case study of west Yangshuo Street. Human Geography, 27(6): 81-86. ]

张景秋, 刘欢, 齐英茜, 等. 2015. 北京城市老年人居住环境 及生活满意度分析 [J]. 地理科学进展, 34(12): 1628-
1636. [Zhang J Q, Liu H, Qi Y Q, et al. 2015. Living environment and life satisfaction of aged population in Beijing Municipality. Progress in Geography, 34(12): 1628-1636. ] 张艳, 柴彦威. 2013. 生活活动空间的郊区化研究 [J]. 地理科 学进展, 32(12): 1723-1731. [Zhang Y, Chai Y W. Study on suburbanization of living and activity space. Progress in Geography, 32(12): 1723-1731. ]

庄春萍, 张建新. 2011. 地方认同:环境心理学视角下的分析 [J]. 心理科学进展, 19(9): 1387-1396. [Zhuang C P, Zhang J X. 2011. Place identity: Analysis from environmental psychology perspective. Advances in Psychological Science, 19(9): 1387-1396. ]

Lewicka M. 2005. Ways to make people active: The role of place attachment, cultural capital, and neighborhood ties [J]. Journal of Environmental Psychology, 25(4): 381-395.

Lu T T, Zhang F Z, Wu F L. 2018. Place attachment in gated neighbourhoods in China: Evidence from Wenzhou $[\mathrm{J}]$. Geoforum, 92: 144-151.

Proshansky H M. 1978. The city and self-identity graduate school and graduate center of the city university of New York [J]. Environment and Behavior, 10: 147-169.

Proshansky H M, Fabian A K, Karminoff R. 1983. Place identity: Physical world socialization of the self [J]. Journal of Environmental Psychology, 3: 57-84.

Qian J X, Zhu H. 2014. Chinese urban migrants' sense of place: Emotional attachment, identity formation, and place dependence in the city and community of Guangzhou [J]. Asia Pacific Viewpoint, 55(1): 81-101.

Qian J X, Zhu H, Liu Y. 2011. Investigating urban migrants' sense of place through a multi-scalar perspective [J]. Journal of Environmental Psychology, 31: 170-183.

Stedman R C. 2006. Understanding place attachment among second home owners [J]. American Behavioral Scientist, 50 (2): 187-205.

Wu R, Li Z G, Liu Y, et al. 2019. Neighborhood governance in post-reform urban China: Place attachment impact on civic engagement in Guangzhou [J]. Land Use Policy, 81: 472-482.

Zhu Y, Breitung W, Li S M. 2011. The changing meaning of neighbourhood attachment in Chinese commodity housing estates: Evidence from Guangzhou [J]. Urban Studies, 49 (11): 2439-2457. 


\title{
Neighborhood attachment of suburban residents in the context of community life circle construction: A case study in Shanghai
}

\author{
FU Xingxing ${ }^{1,2}$, SHEN Yue ${ }^{2,3^{*}}$ \\ (1. School of Architecture and Urban Planning, Nanjing University, Nanjing 210093, China; \\ 2. School of Urban and Regional Science, East China Normal University, Shanghai 200062, China; \\ 3. The Center for Modern Chinese City Studies, East China Normal University, Shanghai 200062, China)
}

\begin{abstract}
Under the background of rapid suburbanization, suburban areas have become the most changeable and complex region in metropolitan areas. Increasing the dependence of residents' daily activities on neighborhood space is of great importance for relieving the pressure of urban development and improving the quality of life of residents. Studies show that residents' psychological dependence on a neighborhood also has a positive impact on life satisfaction. Based on the concept of people- oriented development, Shanghai City Master Plan 20172035 proposes to build 15 -minute life circles, and to optimize the spatial structure of suburbs. In the context of rapid suburbanization and community life circle construction, this research built a framework that combines the two dimensions of functional attachment and emotional attachment to study neighborhood attachment of residents in Shanghai suburbs. It aimed to answer the following research questions: 1) How is residents' neighborhood attachment from a comprehensive perspective that combines the two dimensions of functional attachment and emotional attachment? 2) What are the differences of neighborhood attachment among various social groups? 3) How do the built environment and the subjective perception of neighborhood environments impact individuals' neighborhood attachment? The data used in this research came from an activity- travel behavior survey conducted in Shanghai suburbs in 2017. The data include individuals' socioeconomic attributes, daily activities, and perception of communities. First, this study analyzed the characteristics of residents' neighborhood attachment and the differences of different social groups. Second, on the basis of the research framework, residents' neighborhood attachment was divided into four types: neighborhood attachment, neighborhood dependence, neighborhood identity, and neighborhood exclusion. Multiple Logistics regression models were used to analyze the impacts of the built environment and subjective perception of neighborhood on individuals' neighborhood attachment type after socioeconomic attributes were controlled for. Third, based on the results of regression analyses, we represent the relationships between socioeconomic attributes and neighborhood attachment, and neighborhood characteristics and neighborhood attachment by two coordinate systems. The differences of neighborhood attachment among different social groups are significant. Neighborhood characteristics have significant influences on neighborhood attachment: the density of neighborhood facilities mainly influences functional attachment; neighborhood green space ratio influences both functional attachment and emotional attachment; residents' subjective evaluation of neighborhood environment mainly influences emotional attachment. According to the research findings, the construction of community life circle should maintain and improve environment quality and focus on the arrangement of green space, open space, and leisure facilities. Meanwhile, it is important to promote the social integration of residents in the neighborhoods.
\end{abstract}

Keywords: neighborhood attachment; community life circle; community attachment; Shanghai; suburbs 